\title{
A case of iatrogenic pneumothorax in which chest tube placement could be avoided by intraoperative evaluation with transthoracic ultrasonography
}

\author{
This article was published in the following Dove Press journal: \\ Therapeutics and Clinical Risk Management \\ 5 July 2017 \\ Number of times this article has been viewed
}

\author{
Izumi Sato \\ Hirotsugu Kanda \\ Megumi Kanao-Kanda \\ Atsushi Kurosawa \\ Takayuki Kunisawa \\ Department of Anesthesiology and \\ Critical Care Medicine, Asahikawa \\ Medical University, Asahikawa, \\ Hokkaido, Japan
}

\begin{abstract}
We report a case of iatrogenic pneumothorax in which chest tube placement was avoided by continuous intraoperative evaluation with transthoracic ultrasonography. A 53-yearold man had undergone a subsegmentectomy. While attempting to place a central venous catheter in the right internal jugular vein after the induction of anesthesia, we identified gas absorption during the puncture and suspected a pneumothorax. Chest X-ray revealed an $\sim 5$-mm collapse of the right lung apex. Tension pneumothorax was a concern during surgery because of the long-term positive pressure ventilation, but we decided to start the operation without preventative chest tube placement. During the operation, we regularly observed the midclavicular line of the second intercostal space using ultrasound. The operation was completed uneventfully. In this case, we effectively utilized ultrasound and avoided preventive chest tube placement and the associated complications. Transthoracic ultrasonography could be performed easily and continuously during surgery and was effective for evaluating the progression of an intraoperative pneumothorax.
\end{abstract}

Keywords: transthoracic ultrasonography, iatrogenic pneumothorax, central venous catheterization

\section{Introduction}

Pneumothorax is a complication of central vein catheter insertion. If the pneumothorax is minimal, the initial clinical signs may be subtle and tension pneumothorax could occur with positive pressure ventilation under general anesthesia. Chest X-ray and chest computed tomography (CT) are commonly used to diagnose a pneumothorax, but are difficult to perform frequently and continuously in the operating room. A chest tube is usually inserted to treat a pneumothorax, but chest tubes are invasive and have a risk of complication. We report a case of iatrogenic pneumothorax in which chest tube placement was avoided by continuous evaluation of pneumothorax progression using intraoperative transthoracic ultrasonography.

\section{Consent for publication}

Written and signed informed consent was obtained from the patient for publication of this case report as well as the accompanying image.

\section{Case report}

A 53-year-old man had undergone an open S8 subsegmentectomy of the anterosuperior segment of right hepatic lobe for hepatocellular carcinoma. After inducing anesthesia, 
we attempted to place a central venous catheter in the right internal jugular vein under ultrasound guidance. We suspected a pneumothorax due to an unclear image of the needle tip and gas absorption during the puncture. Auscultation using a stethoscope revealed no lateralized changes in the respiratory sounds, and we observed no changes in the vital signs or aggravation due to hypooxygenation. We performed transthoracic ultrasonography (iE33, Philips; linear probe; Tokyo, Japan) at four points of the chest, the midclavicular line of the second intercostal space, the axillary line in front of the fourth intercostal space, and the middle and posterior axillary lines of the sixth intercostal space. In transthoracic ultrasonography, absent lung sliding (ie, respiratory movement at the pleural line) is a positive sign of pneumothorax. Ultrasound at four points of the chest revealed the lung sliding sign; hence, there were no findings to clearly suggest pneumothorax. On the other hand, chest X-ray revealed a small 5-mm collapse of the right lung apex (Figure 1). Tension pneumothorax during surgery was a concern due to the long-term positive pressure ventilation. We evaluated whether we should place a chest tube preventatively, but decided that the risk of complications, such as damage to the lungs, intercostal veins, arteries, and nerves, as well as subcutaneous emphysema, was too great. We designated an absence of the lung sliding sign at the midclavicular line of the second intercostal space as an indicator of pneumothorax progression. We decided not to place the prophylactic chest tube for urgent deaeration. During the operation, we regularly observed the midclavicular line of the second intercostal space using ultrasound, but there was no evidence to suggest that the pneumothorax was progressing. The operation was completed uneventfully. Postoperative chest X-ray revealed extremely low progression of the pneumothorax. Aggravated breathing was not observed following extubation. Chest X-ray 5 days postoperatively revealed that the pneumothorax had healed, and the patient was discharged on day 10 .

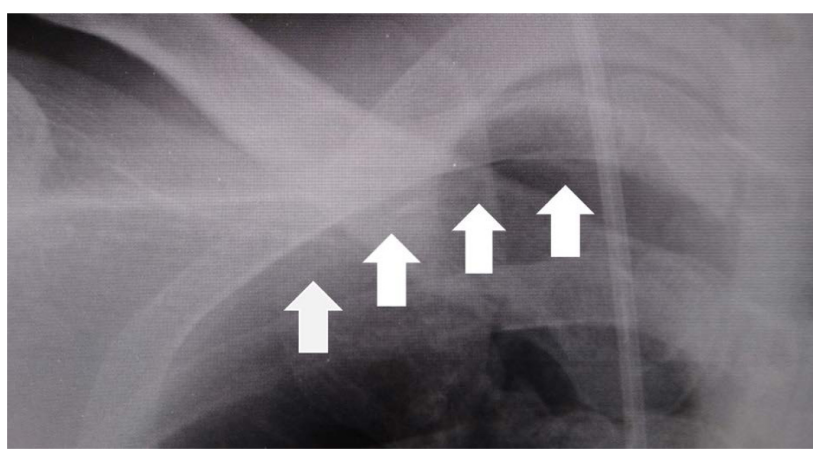

Figure I Chest X-ray film immediately after the puncture. Note: An $\sim 5 \mathrm{~mm}$ collapse of the apical portion of the right lung is observed (arrows).

\section{Discussion}

In some cases, ultrasonography is used to diagnose an intraoperative pneumothorax, which can then be treated by inserting a chest tube. ${ }^{1}$ To the best of our knowledge, this is the first report of monitoring the progression of a minimal pneumothorax by intraoperative ultrasonography under positive pressure ventilation, thus avoiding chest tube placement. The progression of the pneumothorax could be successfully evaluated using ultrasonography because the collapsed area was very small and a functional indicator, the lung sliding sign at the midclavicular line of the second intercostal space, could be visualized.

Ultrasonography is now widely applied for diagnosing traumatic pneumothorax in the field of emergency care. A traumatic pneumothorax can progress to tension pneumothorax, and tension pneumothorax is a condition that can quickly cause death from respiratory and circulatory failure and must therefore be diagnosed immediately. In emergency situations, it must be determined whether immediate deaeration is necessary, and whether positive pressure ventilation can be performed, because it can worsen pneumothorax, in particular, in situations CT cannot be used to establish an exact diagnosis. CT has various limitations, such as transportability, that can delay diagnosis and treatment. Portable $\mathrm{X}$-ray has often been performed in the past, but many studies have reported the utility of ultrasonography to diagnose pneumothorax. Although the sensitivity ranges widely, several studies demonstrated that ultrasonography was more sensitive than supine X-ray. ${ }^{2-5}$ In the current case, transthoracic ultrasonography did not diagnose the pneumothorax although chest X-ray detected the change in the right lung. The reason for this was the very small area of the pneumothorax in the pulmonary apex, which was obscured by the clavicle. We recommend combined evaluation using ultrasonography and X-ray to accurately diagnose pneumothorax when a pneumothorax is suspected intraoperatively.

In the cases of a minimal pneumothorax detected by intraoperative chest X-ray, it is not clear whether the anesthesiologist should insert a chest tube. Previously, when pneumothorax was discovered in a patient undergoing positive pressure ventilation, it was believed that a chest tube should be inserted for preventive purposes. ${ }^{6,7}$ On the other hand, several recent reports indicated that observation of an occult pneumothorax observed by CT, but not chest X-ray, in patients on positive pressure ventilation is not associated with an increased incidence of pneumothorax progression or respiratory distress, indicating that an occult pneumothorax could be safely observed in patients under positive pressure ventilation. ${ }^{8-10}$ 
According to the British Thoracic Society guideline, a chest tube is not required if the distance from the apex to the cupola is less than $2 \mathrm{~cm} .{ }^{11}$ In the current case, the distance from the apex to the cupola was only $5 \mathrm{~mm}$, and therefore we decided not to insert a chest tube to avoid the associated complications. We could manage the minimal pneumothorax diagnosed by the chest X-ray safely without placing a chest tube by monitoring the progression of the pneumothorax using thoracic ultrasonography.

There are several reports showing the usefulness of transthoracic ultrasonography in operating rooms, ${ }^{1,12}$ where it is difficult to frequently obtain chest X-rays and CT scans, and there are also several reports that the progression of pneumothorax on transthoracic ultrasonography correlates with that on CT. ${ }^{13-16}$ In the current case, evaluation of the midclavicular line of the second intercostal space using ultrasonography was considered sufficient to monitor the progression of the pneumothorax. Intraoperative ultrasonography did not detect the absence of lung sliding - respiratory movement at the pleural line - on the midclavicular line of the second intercostal space. An X-ray obtained after the operation also showed no clear progression of the pneumothorax. In other words, in the operating room and the intensive care unit, ultrasonography can be an effective tool for evaluating the progression of a pneumothorax, facilitating the decision about whether chest tube placement is necessary for traumatic or iatrogenic pneumothorax with positive pressure ventilation.

\section{Conclusion}

In this case, the effective use of ultrasonography avoided preventive chest tube placement and the associated complications. Transthoracic ultrasonography can be easily and continuously performed during surgery and was effective for monitoring the progression of an intraoperative pneumothorax.

\section{Disclosure}

The authors report no conflicts of interest in this work.

\section{References}

1. Ueda K, Ahmed W, Ross AF. Intraoperative pneumothorax identified with transthoracic ultrasound. Anesthesiology. 2011;115(3):653-655.

2. Ali V, Hamid RH, Kamran H, Hosein A, Mohammad T. Diagnostic accuracy of ultrasonography and radiography in initial evaluation of chest trauma patients. Emergency. 2016;4(1):29-33.

3. Brook OR, Beck-Razi N, Abadi S, et al. Sonographic detection of pneumothorax by radiology residents as part of extended focused assessment with sonography for trauma. J Ultrasound Med. 2009;28(6): 749-755.

4. Ianniello S, Di Giacomo V, Sessa B, Miele V. First-line sonographic diagnosis of pneumothorax in major trauma: accuracy of e-FAST and comparison with multidetector computed tomography. Radiol Med. 2014;119(9):674-680.

5. Arajhi K, Woo MY, Vaillancourt C. Test characteristics of ultrasonography for the detection of pneumothorax: a systematic review and meta-analysis. Chest. 2012;141(3):703-708.

6. American College of Surgeons. American College of Surgeons Committee on Trauma: Advanced Trauma Life Support Course for Doctors. Instructors Course Manual bth ed. American College of Surgeons. Chicago 1997.

7. Enderson BL, Abdalla R, Frame SB, Casey MT, Gould H, Maull KI Tube thoracostomy for occult pneumothorax: a prospective randomized study of its use. J Trauma. 1993;35(5):726-729.

8. Brasel KJ, Stafford RE, Weigelt JA, Tenquist JE, Borgstrom DC Treatment of occult pneumothoraces from blunt trauma. $J$ Trauma. 1999;46(6):987-991.

9. Ouellet JF, Trottier V, Kmet L, et al. The OPTICC trial: a multiinstitutional study of occult pneumothoraces in critical care. Am J Surg. 2009;197(5):581-586.

10. Kirkpatrick AW, Rizoli S, Ouellet JF, et al; Canadian Trauma Trials Collaborative and the Research Committee of the Trauma Association of Canada. Occult pneumothoraces in critical care. $J$ Trauma Acute Care Surg. 2013;74(3):747-755.

11. MacDuff A, Arnold A, Harvey J; BTS Pleural Disease Guideline Group. Management of spontaneous pneumothorax: British Thoracic Society Pleural Disease Guideline 2010. Thorax. 2010;65(suppl 2):ii18-ii31.

12. Johnson DW, Oren-Grinberg A. Perioperative point-of-care ultrasonography: the past and the future are in anesthesiologists' hands. Anesthesiology. 2011;115(3):460-462.

13. Soldati G, Testa A, Sher S, Pignataro G, La Sala M, Silveri NG. Occult traumatic pneumothorax: diagnostic accuracy of lung ultrasonography in the emergency department. Chest. 2008;133(1):204-211.

14. Zhang M, Liu Z-H, Yang J-X, et al. Rapid detection of pneumothorax by ultrasonography in patients with multiple trauma. Crit Care. 2006;10(4):R112.

15. Blaivas M, Lyon M, Duggal S. A prospective comparison of supine chest radiography and bedside ultrasound for the diagnosis of traumatic pneumothorax. Acad Emerg Med. 2005;12(9):844-849.

16. Soldati G, Testa A, Pignataro G, et al. The ultrasonographic deep sulcus sign in traumatic pneumothorax. Ultrasound Med Biol. 2006; 32(8):1157-1163.
Therapeutics and Clinical Risk Management

\section{Publish your work in this journal}

Therapeutics and Clinical Risk Management is an international, peerreviewed journal of clinical therapeutics and risk management, focusing on concise rapid reporting of clinical studies in all therapeutic areas, outcomes, safety, and programs for the effective, safe, and sustained use of medicines. This journal is indexed on PubMed Central, CAS,

\section{Dovepress}

EMBase, Scopus and the Elsevier Bibliographic databases. The manuscript management system is completely online and includes a very quick and fair peer-review system, which is all easy to use. Visit http://www.dovepress.com/testimonials.php to read real quotes from published authors. 